\title{
Inhibition of RAD54B suppresses proliferation and promotes apoptosis in hepatoma cells
}

\author{
RUI WANG ${ }^{1,2^{*}}$, YAWEI LI $^{2 *}$, YUFO CHEN ${ }^{2}$, LEI WANG ${ }^{1}$, QIONG WU $^{2}$, \\ YUANYUAN GUO ${ }^{3}$, YUMEI LI $^{2}$, JING LIU ${ }^{2}$ and LIWEI WANG ${ }^{1}$ \\ ${ }^{1}$ Department of Oncology, Shanghai General Hospital of Nanjing Medical University, \\ Shanghai 201620; Departments of ${ }^{2}$ Medical Oncology and ${ }^{3}$ Urology, First Affiliated Hospital \\ of Bengbu Medical College, Bengbu, Anhui 233004, P.R. China
}

Received January 15, 2018; Accepted June 15, 2018

DOI: $10.3892 /$ or.2018.6522

\begin{abstract}
RAD54 homolog B is a central motor protein of homologous recombination (HR), which plays an important role in the development and evolution of human cancer. Recent studies have indicated that RAD54B is aberrantly expressed in a variety of cancers and exhibits numerous biological functions, including participating in the repair of DNA double strand breaks. However, the expression and function of RAD54B in liver cancer have not yet been reported. The aim of the present study was to investigate the expression of RAD54B and elucidate its role in liver cancer cell lines by inhibiting RAD54B using a lentivirus-mediated shRNA interference system. We also assessed the effect of RAD54B on cell proliferation, colony formation, cell cycle distribution and cell apoptosis in BEL-7404 and SMMC-7721 cell lines using shRAD54B or shCtrl transfection. Furthermore, we analyzed the relationship between the expression of RAD54B protein, as measured by immunohistochemical staining, and the prognosis of patients with hepatoma. We found that RAD54B was highly expressed in liver cancer cell lines compared with the normal hepatic cell line LO2. Similarly, positive expression of RAD54B, which is associated with poor prognosis, was also observed in 52/83 samples of liver cancer tissue. Additionally, RAD54B downregulation significantly inhibited cell proliferation and colony formation, while also inducing G1/S cell cycle arrest and apoptosis in BEL-7404 and SMMC-7721 cells. These results indicated that RAD54B has oncogenic properties, and may be a potential treatment target for liver cancer patients.
\end{abstract}

Correspondence to: Dr Liwei Wang, Department of Oncology, Shanghai General Hospital of Nanjing Medical University, 650 Xin Songjiang Road, Shanghai 201620, P.R. China

E-mail: liweiwang@shsmu.edu.cn

*Contributed equally

Key words: liver cancer, RAD54B, homologous recombination, cell growth, cell apoptosis

\section{Introduction}

Primary liver cancer is a common aggressive malignant disease, and the third leading cause of cancer-related deaths worldwide. An estimated 40,710 new cases of disease and 28,920 cancer-associated deaths were reported in 2017 in the USA (1). More than $90 \%$ of primary liver cancers are hepatoma, which is mainly caused by infection with hepatitis B or $\mathrm{C}$. The incidence of liver cancer has been increasing in Asia and $~ 50 \%$ of reported mortalities are Chinese individuals (2). As early detection is difficult, $\sim 70 \%$ of all hepatoma patients cannot be treated by surgical resection at the time of diagnosis, which results in a poor prognosis and a median overall survival time of a few months (3). The molecular pathogenesis of liver cancer has been extensively investigated in recent years; however, no effective biomarker for the early diagnosis and treatment of hepatoma has been established.

Emerging evidence has demonstrated that abnormal homologous recombination repair (HRR) is closely related to the development and progression of human cancers (4). The function of proteins that participate in homologous recombination (HR) is primarily to maintain genomic stability and suppress tumor development by repairing DNA double strand breaks (DSBs) and damaged replication forks $(5,6)$. RAD51 is a central member of the HRR signaling pathway, which is expressed in a number of cancer cell lines and is associated with cell sensitivity to DNA-damaging agents $(7,8)$. RAD51associated protein 1 (RAD51AP1) mRNA expression is increased in liver cancer tissues compared with corresponding normal liver tissues (9). Obama et al revealed that RAD51AP1 expression was increased in intrahepatic cholangiocarcinoma, and the downregulation of RAD51AP1 by shRNA could effectively suppress the proliferation of cholangiocarcinoma cells (10). Recent findings have revealed that RAD52 may be a potential therapeutic target for BRCA1 and BRCA2-deficient familial breast and ovarian cancer (11-13). It has also been demonstrated that disease-free survival is correlated with RAD50 expression in tissues from patients with non-small cell lung cancer (NSCLC); RAD50 knockdown increases cell sensitivity to radiation whereas RAD50 upregulation induces radioresistence in NSCLC cells (14). These previous studies indicated that many HR-associated proteins are involved in 
the development of cancers, including liver cancer; however, there are still many HRR-associated proteins that regulate the growth and function of cancer cells and need to be further elucidated.

Recently, our group investigated RAD54B, which is a vital motor protein of HR. RAD54B belongs to the SNF2/SWI2 superfamily and plays an important role in the DNA repair system. It has been revealed that distant metastasis was significantly increased in colorectal cancer patients with high RAD54B expression compared with the low expression group, which may be associated with the degradation of $\mathrm{p} 53$ protein in clinical samples (15). In addition, the high expression of Rad54B may act as an independent prognostic factor for lung adenocarcinoma (16). To the best of our knowledge, few studies have reported the effect of RAD54B on the development of cancer and the mechanism by which it functions. Furthermore, there have been no previous reports regarding the expression and biological function of RAD54B in liver cancer.

In the present study, we investigated the expression of RAD54B in liver cancer and analyzed its relationship with liver cancer patient prognosis. Furthermore, we explored the effect of RAD54B silencing on hepatoma cell proliferation, colony formation, cell cycle distribution and apoptosis. This study aimed to identify a potential new biomarker or treatment target that could be used for the prognosis of liver cancer patients.

\section{Materials and methods}

Gene expression profiles. RAD54B mRNA expression data from 50 liver cancer tissues and 50 matched adjacent tissue samples were obtained from the TCGA data portal (https:// tcga-data.nci.nih.gov/tcga/).

Human tissue samples, cell culture, reagents and antibodies. The human tissue protocol utilized in this study was approved by the Ethics Committee of Bengbu Medical College. A total of 83 samples were obtained from patients with liver cancer who underwent surgery at the First Affiliated Hospital of Bengbu Medical College (Bengbu, China) between January 2012 and November 2015. Preoperative informed consent was obtained from each patient registered in the study, in accordance with the institutional guidelines. Harvested specimens were subjected to immunohistochemistry (IHC). Human LO2, BEL-7404, BEL-7402, HepG2 and SMMC-7721 cell lines were purchased from the Cell Bank of the Shanghai Institute of Cell Biology, Chinese Academy of Sciences (Shanghai, China). The cells were cultured at $37^{\circ} \mathrm{C}$ in $5 \% \mathrm{CO}_{2}$ in Dulbecco's modified Eagle's medium (DMEM; Corning, Inc., Corning, NY, USA) containing $10 \%$ fetal bovine serum (FBS; Gibco; Thermo Fisher Scientific, Inc., Waltham, MA, USA). MTT [3-(4,5-dimethythiazol- 2-yl)-2,5-diphenyl tetrazolium bromide] was purchased from Genview Scientific, Inc. (El Monte, CA, USA). An Annexin V Apoptosis Detection kit was obtained from BD Biosciences (San Diego, CA, USA). Rabbit polyclonal anti-RAD54B antibodies (cat. no. ab83311) and mouse anti- $\beta$-actin monoclonal antibodies (cat. no. ab8226) were obtained from Abcam (Cambridge, MA, USA).

Immunohistochemistry (IHC). Formalin-fixed and paraffinembedded liver cancer tissue samples were cut into $4-\mu$ m-thick slices. After deparaffinization, $3 \% \mathrm{H}_{2} \mathrm{O}_{2}$ was used for 15 min to block the endogenous peroxidase and reduce non-specific staining. Antigen retrieval was then performed with sodium citrate buffer ( $\mathrm{pH}$ 6.0). The slices were incubated with primary antibodies (rabbit anti-RAD54B polyclonal antibodies, dilution 1:50; Abcam) overnight at $4^{\circ} \mathrm{C}$. After washing, the slices were incubated for $1 \mathrm{~h}$ at $37^{\circ} \mathrm{C}$ with the goat anti-rabbit $\mathrm{IgG}$ second antibodies (dilution 1:1,000; cat. no. ab6720; Abcam). The sections were treated using the SABC kit (Wuhan Boster Biological Technology, Ltd., Wuhan, China) according to the manufacturer's instructions and stained using a 3,3'-diaminobenzidine tetrahydrochloride (DAB) substrate kit. Normal rabbit serum was used in place of the primary antibody as the negative control. Representative images were visualized using an Olympus inverted microscope (Olympus Corp., Tokyo, Japan). Staining was evaluated and scored by two independent researchers. The percentage ratio of positively stained cells vs. all cells was calculated in 10 randomly selected microscopic fields at magnification $\mathrm{x} 400$. Staining localized to the nucleus was graded on a 0-3 intensity scale ( 0 , negative; 1 , weakly positive; 2 , moderately positive; 3 , strongly positive). Positive staining was defined as $>5 \%$ of the tumor cells being stained by the antibodies.

Real-time quantitative reverse transcription-PCR. Total RNA from the liver cancer cells was extracted using TRIzol (Invitrogen; Thermo Fisher Scientific, Inc.), which was then treated with an RNeasy Mini kit and RNase-free DNase Set (Promega Corp., Madison, WI, USA) according to the manufacturer's protocols. The primers used in the PCR reactions were as follows: RAD54B forward, 5'-GCCAAACACTG ATGATTTGTGG-3' and reverse, 5'-CCTGAGAAGAATG CGAGATAGC-3'; GAPDH forward, 5'-TGACTTCAACAG CGACACCCA-3' and reverse, 5'-CACCCTGTTGCTGTA GCCAAA-3'. GAPDH was used as the internal control. The fold amplification for gene expression was determined using the $2^{-\Delta \Delta \mathrm{Cq}}$ method (17).

Western blot analysis. Harvested hepatoma cells were washed with phosphate-buffered saline (PBS) and lysed using a RIPA lysis buffer (Wuhan Boster Biological Technology, Ltd.). The concentrations of protein were detected using a Bio-Rad protein assay kit (Bio-Rad Laboratories, Inc., Hercules, CA, USA). Equal amounts of total protein were separated by $10 \%$ sodium dodecyl sulfate-polyacrylamide gel electrophoresis (SDS-PAGE) and transferred to polyvinylidene difluoride (PVDF) membranes. The membranes were blocked with 5\% skimmed milk-TBST and incubated with primary antibodies against RAD54B (1:500; Abcam) at $4^{\circ} \mathrm{C}$ overnight. The membranes were washed three times using TBST before being incubated with HRP-conjugated secondary antibodies (dilution 1:2000; cat. no. ab6721; Abcam) at room temperature for $1 \mathrm{~h}$. The membranes were then washed three times with TBST and proteins were visualized using an electrochemiluminescence (ECL) assay. Images were taken by fusion-capture software (Fusion FX7; Vilber Lourmat, Marne Le-Vallée, France).

Lentivirus infection assay. The targeting sequences of RAD54B (AGATTGTTGATGGCTTTAA) and a nonsense sequence (TTCTCCGAACGTGTTCACGT) were designed 
A

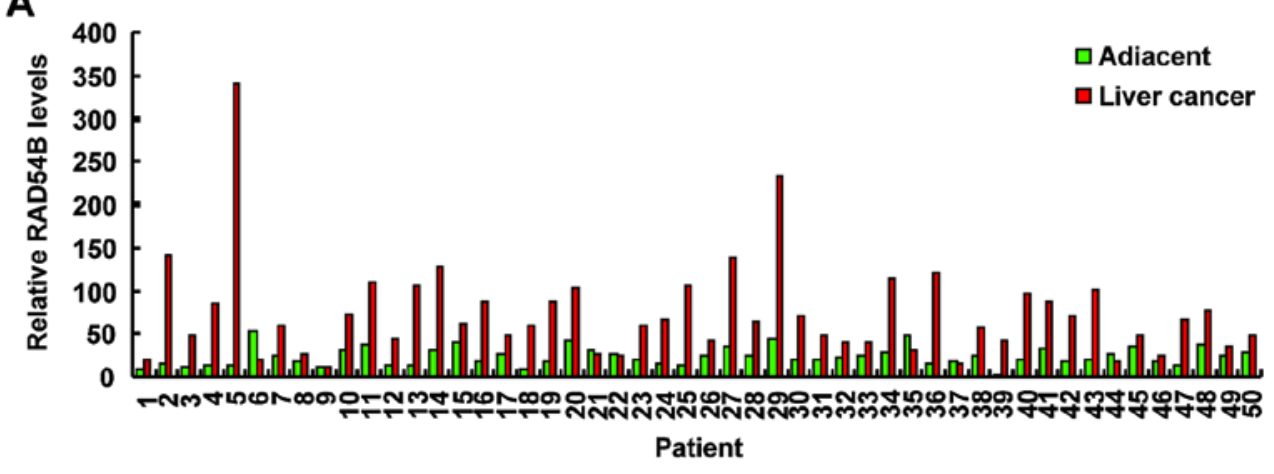

B

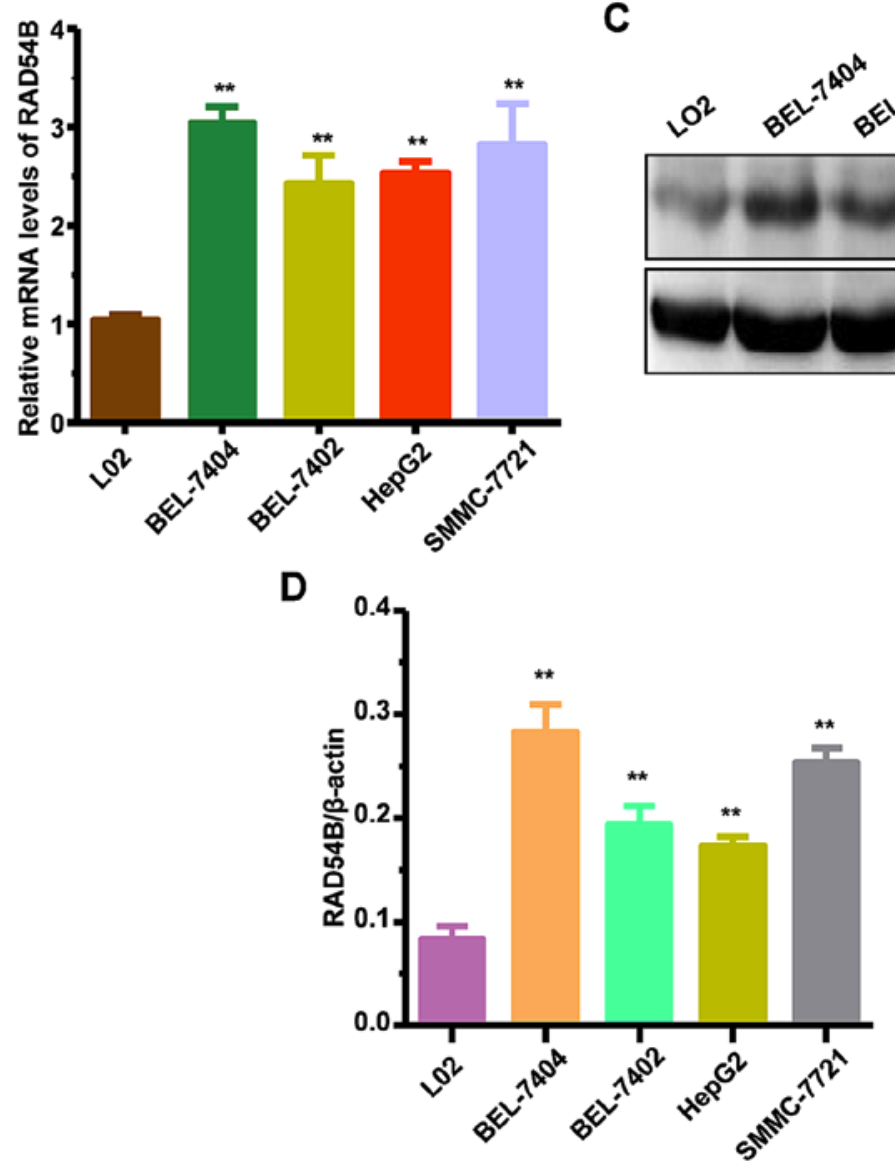

Figure 1. RAD54B expression is increased in liver cancer tissues and cells. (A) RAD54B mRNA expression was analyzed in liver cancer tissues and matched adjacent tissue samples by TCGA database analysis ( $\mathrm{n}=50$, respectively). (B) RT-qPCR was used to detect the RAD54B mRNA expression in the liver cancer cell lines BEL-7404, BEL-7402, HepG2 and SMMC-7721, and the normal hepatic cell line LO2 ( $\left.\mathrm{n}=3,{ }^{* *} \mathrm{P}<0.01\right)$. (C and D) Western blot analysis was performed to determine RAD54B protein expression in the liver cancer cell lines BEL-7404, BEL-7402, HepG2 and SMMC-7721, and the normal hepatic cell line LO2 $\left(\mathrm{n}=3,{ }^{* *} \mathrm{P}<0.01\right)$.

and constructed. RAD54B-shRNA and control-shRNA were inserted into linearized GV115 plasmid vectors (Shanghai GeneChem, Co., Ltd., Shanghai, China), which carried the green fluorescent protein (GFP) gene and U6-MCS-CMVpuror. The RAD54B and control shRNA vectors and two packaging auxiliary plasmids (pHelper1.0 and pHelper2.0) were used for lentivirus production. The plasmid vectors were transformed Escherichia coli competent cells. The positive colonies were selected and identified by PCR and sequencing. Next, the effective plasmid vectors were extracted and packed into lentivirus particles using $293 \mathrm{~T}$ cells. BEL-7404 and SMMC-7721 cells were transfected with the RAD54B-shRNA-lentivirus (shRAD54B group) or negative control lentivirus (shCtrl group) at the recommended multiplicity of infection (MOI) when the cells reached $80 \%$ confluence in the 6 -well plates. Fluorescence expression was observed under a fluorescence microscope (Olympus Corp.) 3 days after lentiviral infection.

MTT assay. BEL-7404 and SMMC-7721 cells were seeded at equal densities in 96-well culture plates and incubated overnight at $37^{\circ} \mathrm{C}$ in $5 \% \mathrm{CO}_{2}$. The cells were incubated with a medium containing $5 \mathrm{mg} / \mathrm{ml}$ MTT for $4 \mathrm{~h}$, following which the medium solution was removed and $100 \mu$ l dimethyl sulfoxide 
A

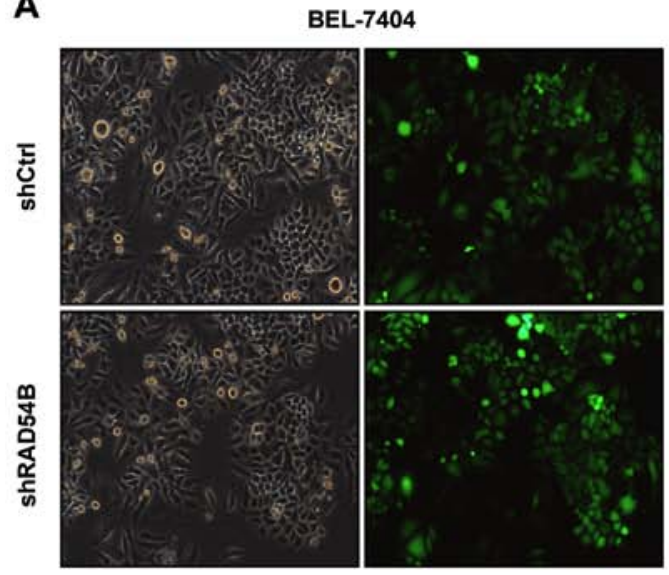

C

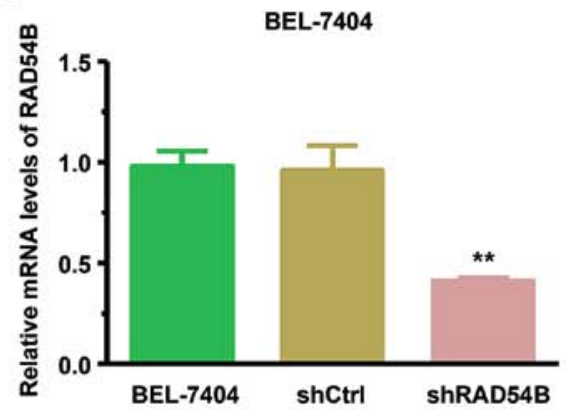

E

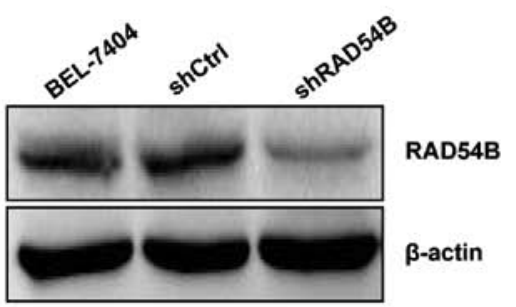

G

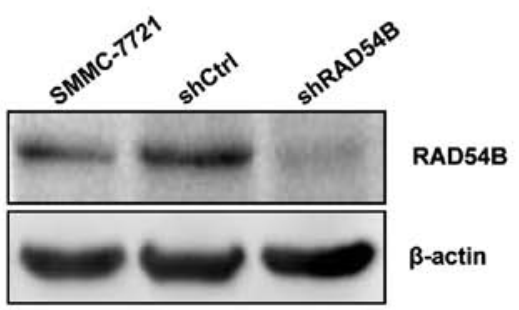

B

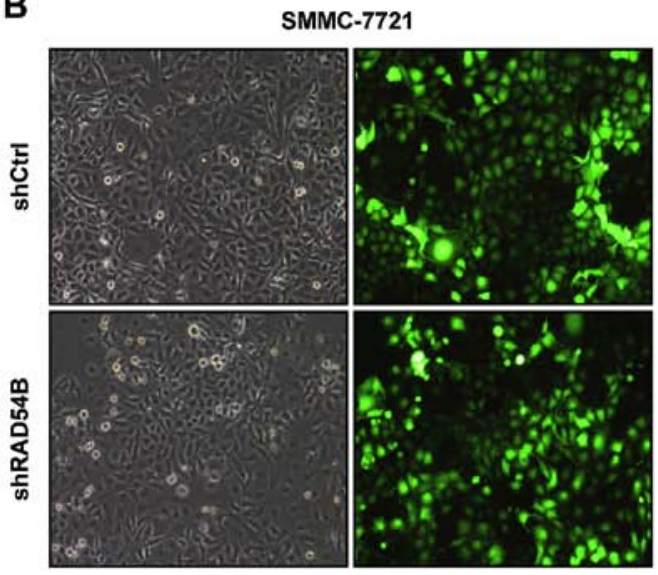

D
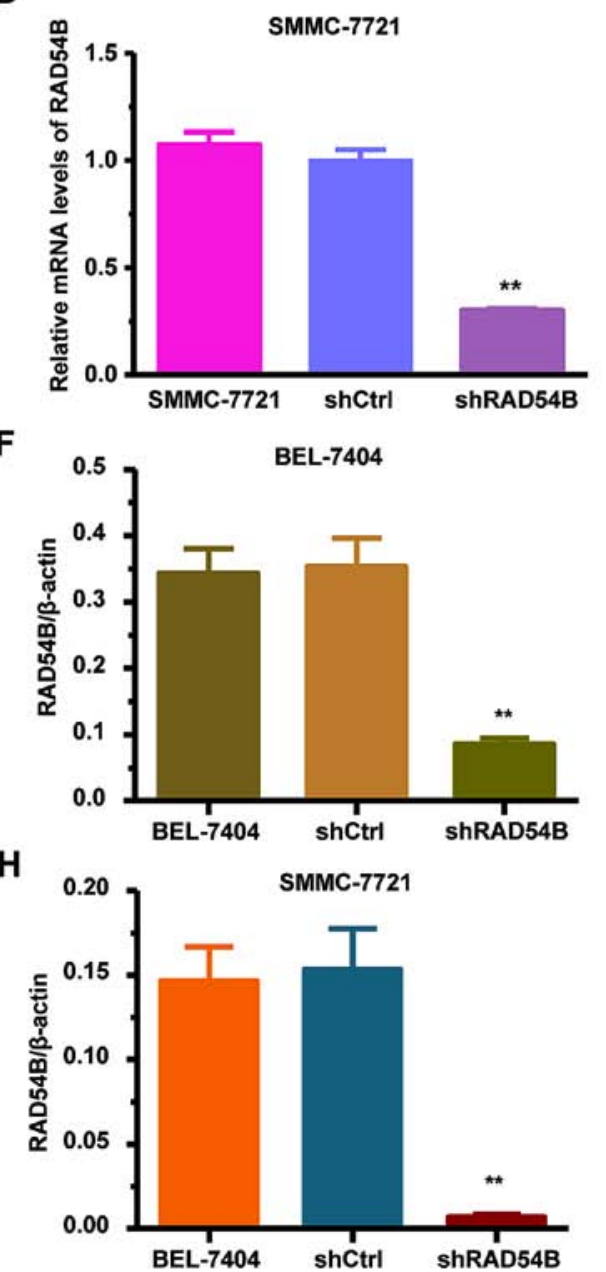

Figure 2. RAD54B mRNA and protein expression are inhibited following infection with shRAD54B in BEL-7404 and SMMC-7721 cells. (A and B) Infection efficiency of lentivirus-shRAD54B (shRAD54B) and lentivirus-shCtrl (shCtrl) in the human hepatoma cell lines BEL-7404 and SMMC-7721. Representative fluorescence images after $72 \mathrm{~h}$ infection are shown (magnification, $\mathrm{x} 100$ ). (C and D) The expression of RAD54B mRNA was analyzed by RT-qPCR after infection with shRAD54B and shCtrl in BEL-7404 and SMMC-7721 cells $\left(n=3,{ }^{* *} \mathrm{P}<0.01\right)$. (E-H) The protein expression of RAD54B was analyzed by western blotting after infection with shRAD54B and shCtrl in BEL-7404 and SMMC-7721 cells and quantification of the results was performed $\left(\mathrm{n}=3,{ }^{* * *} \mathrm{P}<0.01\right)$.

(DMSO) was added to the plates to dissolve the precipitated formazan. The absorbance at $450 \mathrm{~nm}$ was detected using an ELISA microplate reader (Tecan infinite).

Cell proliferation assay. After infection with shRAD54B or shCtrl, the BEL-7404 and SMMC-7721 cells were seeded at a density of 1,000 cells/well in 96-well plates, and then cultured at $37^{\circ} \mathrm{C}$ in $5 \% \mathrm{CO}_{2}$. The Celigo Imaging Cytometry System (Nexcelom Bioscience, Lawrence, MA, USA) was used to observe the fluorescence intensity and calculate the corresponding cell count.

Colony formation assay. After lentiviral infection, the BEL-7404 and SMMC-7721 cells were seeded at a density of 
A

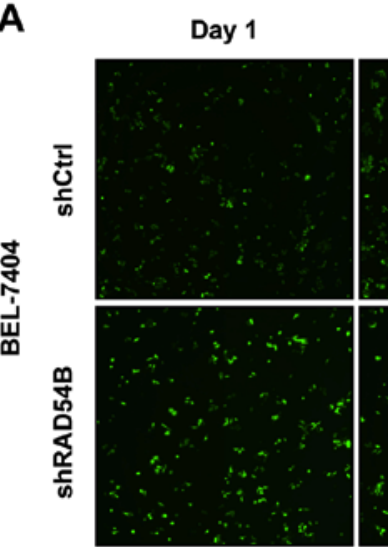

Day 2

Day 3

Day 4

Day 5

B

Day 1
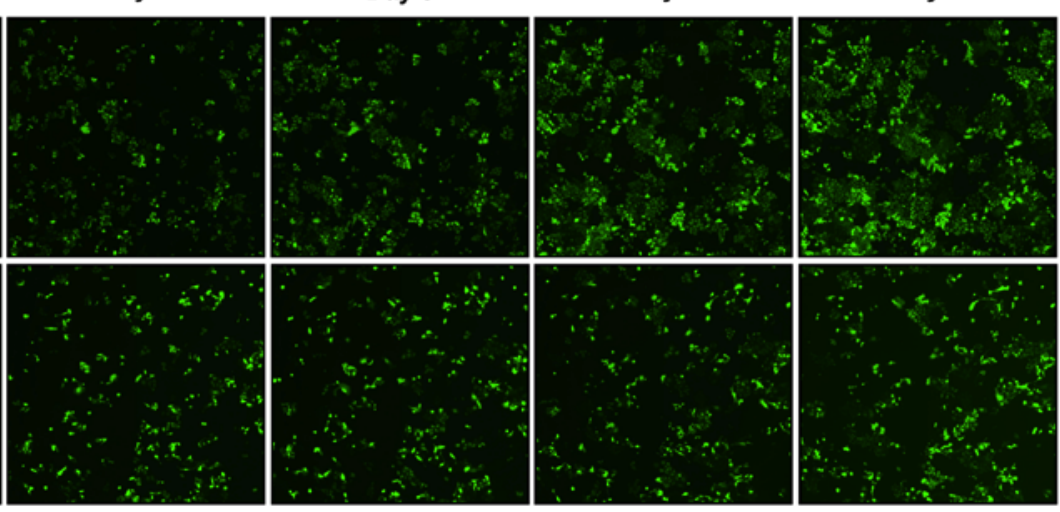

B
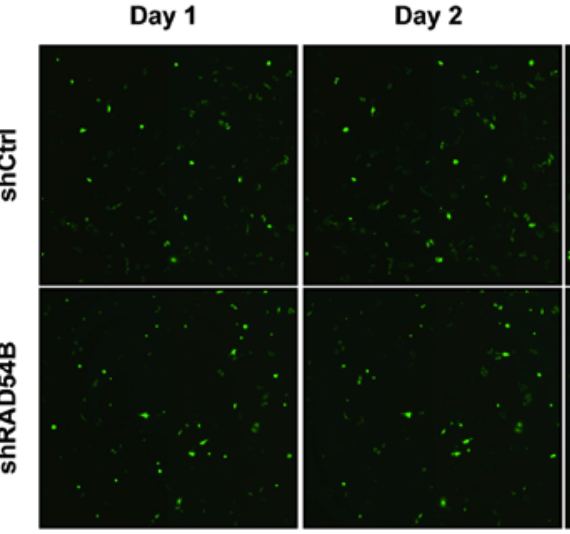

Day 3

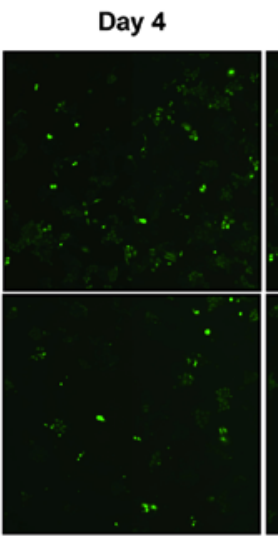

Day 5

C

BEL-7404

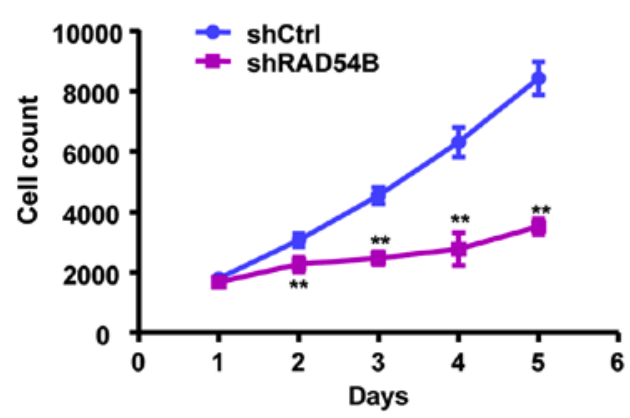

D

SMMC-7721

E

BEL-7404

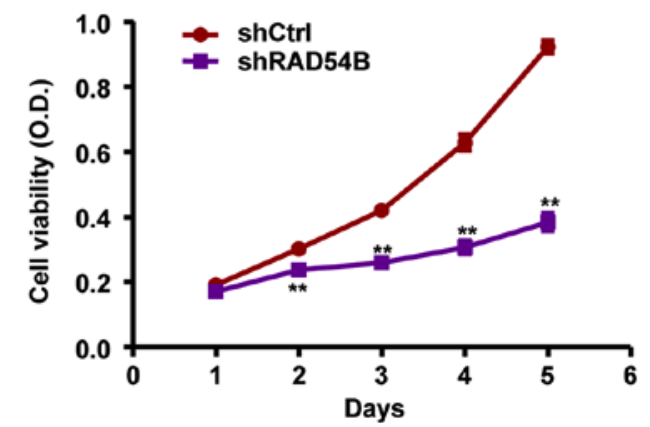

F

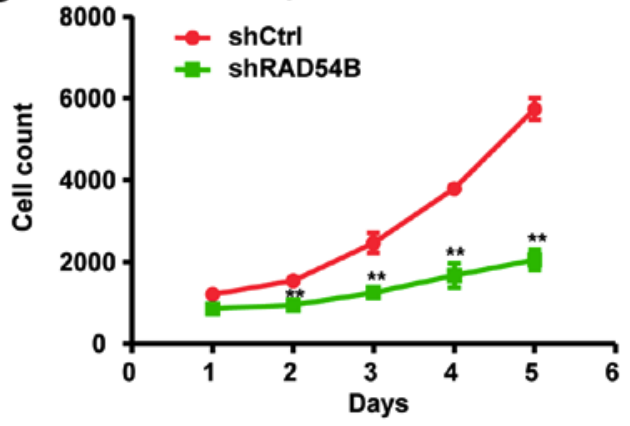

SMMC-7721

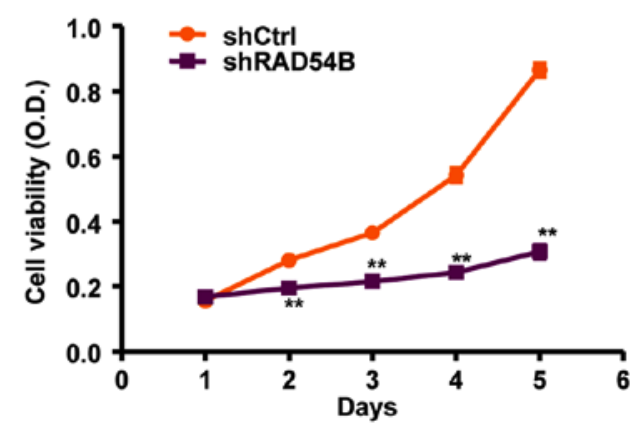

Figure 3. Silencing of RAD54B inhibits proliferation in BEL-7404 and SMMC-7721 cells. (A and B) The Celigo Imaging Cytometry System was used to count the number of cells infected by shCtrl and shRAD54B in the BEL-7404 and SMMC-7721 cell lines from days 1 to 5. Representative fluorescence images are presented (magnification, $\mathrm{x} 100$ ). (C and D) Cell counting statistical curves were constructed according to the Celigo calculations. (E and F) An MTT assay was performed to analyze the proliferation of BEL-7404 and SMMC-7721 cells infected by shCtrl and shRAD54B $\left(\mathrm{n}=3,{ }^{* *} \mathrm{P}<0.01\right)$.

1,000 cells/well in 6-well plates with three duplicates of each and cultured under standard conditions. After 2 weeks, the cells were washed with PBS, stained with Giemsa for $30 \mathrm{~min}$, and washed three times with deionized distilled water to remove any background interference. The number of colonies was counted and images were obtained using a digital camera under light microscopy. The assay was performed independently a minimum of three times. 
A

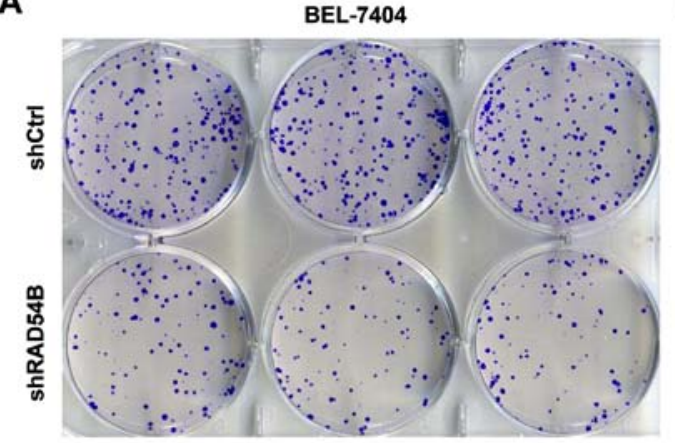

C

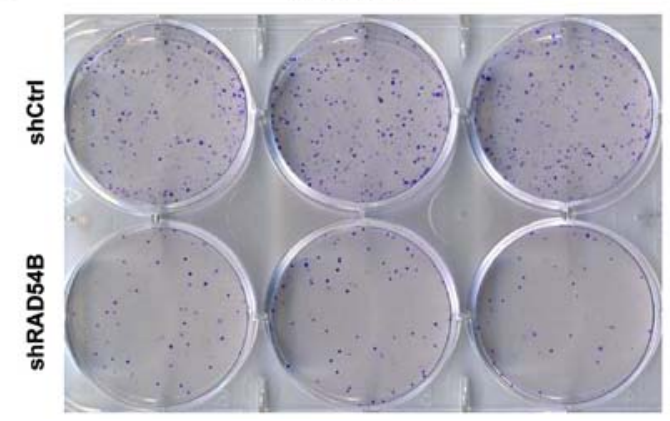

B

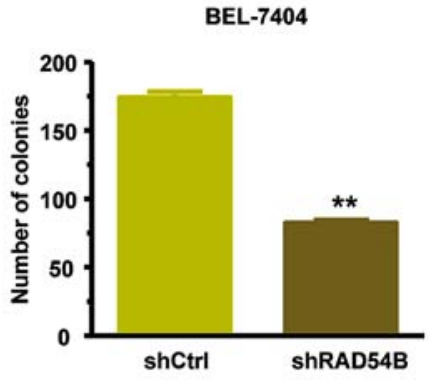

D

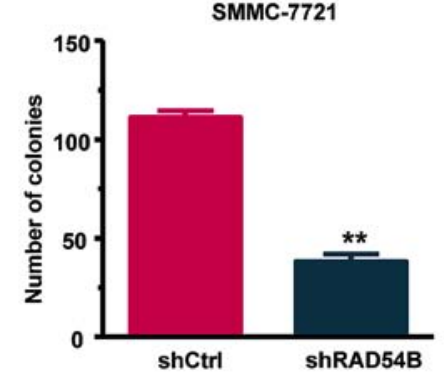

Figure 4. RAD54B downregulation decreases the colony formation abilities of hepatoma cells. (A and C) At 2 weeks after infection with shRAD54B or shCtrl, the BEL-7404 and SMMC-7721 cells were stained with Giemsa and colony formation was observed. Representative images are presented. (B and D) The colony numbers of infected BEL-7404 and SMMC-7721 cells are shown as bar graphs $\left(\mathrm{n}=3,{ }^{* *} \mathrm{P}<0.01\right)$.

Cell cycle distribution analysis. For the cell cycle assay, BEL-7404 and SMMC-7721 cells were seeded into 6-well plates and transfected with the shRAD54B or shCtrl vectors. After the cells were washed with PBS and digested by trypsin, the corresponding dyes and solution were added from the cell cycle kit and incubated according to the manufacturer's instructions. The cell cycle assay was performed using a flow cytometer (EMD Millipore, Billerica, MA, USA) and FlowJo software (FlowJo, LLC, Ashland, OR, USA) was used for analysis.

Apoptosis analysis. The apoptosis rate of BEL-7404 and SMMC-7721 cells infected with shRAD54B or shCtrl was evaluated by Annexin-V PE and 7-AAD staining, using an Apoptosis Detection kit (BD Biosciences, San Diego, CA, USA) according to manufacturer's instructions. Quantification and analysis of the apoptotic cells was performed by flow cytometry (EMD Millipore) and FlowJo software (FlowJo, LLC), respectively.

Statistical analysis. The Student's t-test was performed to estimate the statistical significance of the data. All statistical analysis was performed using SPSS 19.0 software (IBM Corp., Armonk, NY, USA). Data were presented as the mean \pm standard deviation (SD). Kalpan-Meier survival analysis was adopted for overall survival rate analysis. $\mathrm{P}<0.05$ was considered to indicate a statistically significant difference.

\section{Results}

RAD54B expression is elevated in liver cancer tissues and cells. To explore the expression of RAD54B in liver cancer tissues, we collected results from a TCGA dataset. It was found that RAD54B mRNA expression was increased by 2-3-fold in liver cancer tissues compared with paired adjacent tissues (Fig. 1A). Furthermore, we assessed RAD54B mRNA and protein expression in the liver cancer cell lines BEL-7404, BEL-7402, HepG2 and SMMC-7721, and the normal hepatic cell line LO2 using RT-qPCR and western blotting, respectively. The results revealed that RAD54B mRNA and protein expression were both significantly increased in all four liver cancer cell lines compared with the normal LO2 cell line (Fig. 1B-D). These results revealed that RAD54B mRNA and protein expression was elevated in liver cancer tissues and cell lines.

Downregulation of $R A D 54 B$ inhibits the proliferation of BEL-7404 and SMMC-7721 cells. To further investigate the effect of RAD54B on the proliferation of hepatoma cells, we inhibited the expression of RAD54B in the BEL-7404 and SMMC-7721 cell lines. The cells were infected with lentivirusshRAD54B (shRAD54B) and the changes to cell proliferation were observed. Lentivirus-shCtrl (shCtrl) infected cells were used as the control. We observed that $>80 \%$ of the cells were infected by shRAD54B or the shCtrl, which was confirmed by fluorescence microscopy (Fig. 2A and B). The mRNA and protein expression of RAD54B were obviously suppressed in cells infected by shRAD54B compared with those cells infected with shCtrl, as determined by RT-qPCR and western blot analysis (Fig. 2C-H).

In addition, Celigo calculations and an MTT assay were used to determine the effect of RAD54B inhibition on the proliferation of infected cells. The results of the Celigo calculation revealed that the fluorescence intensities of the 
A

BEL-7404

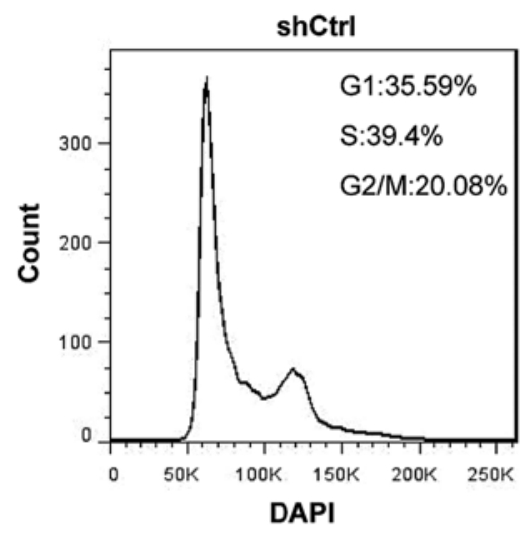

B

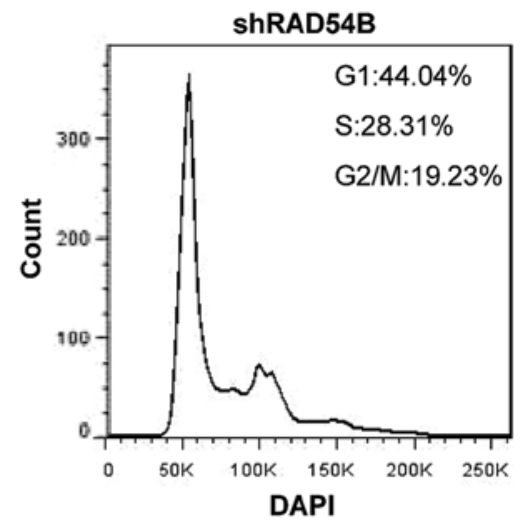

SMMC-7721
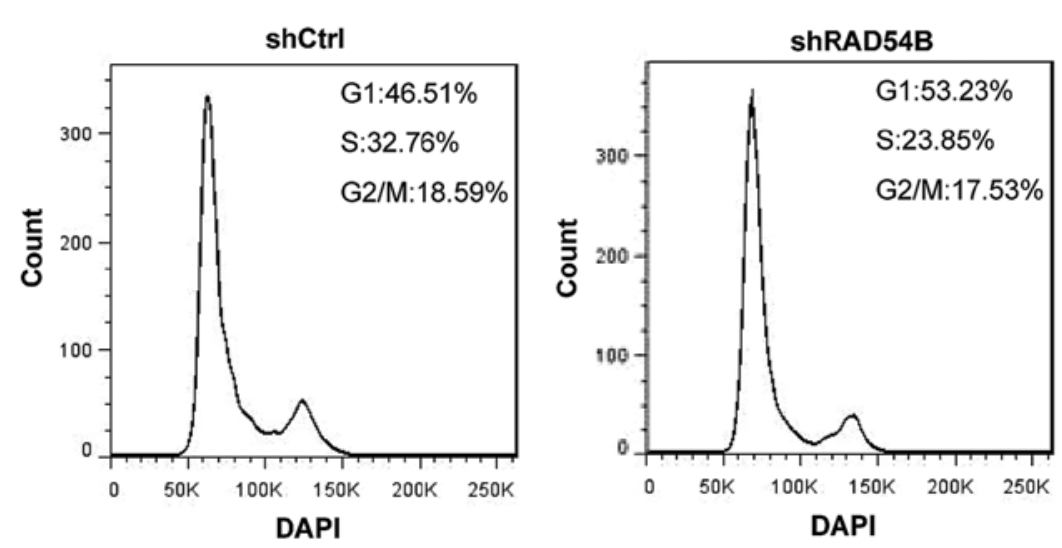

BEL-7404

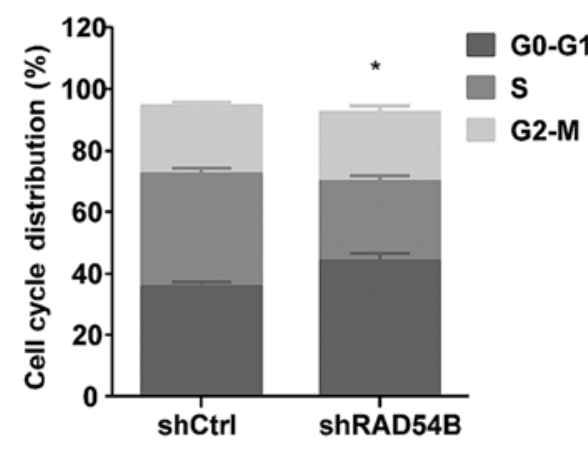

D

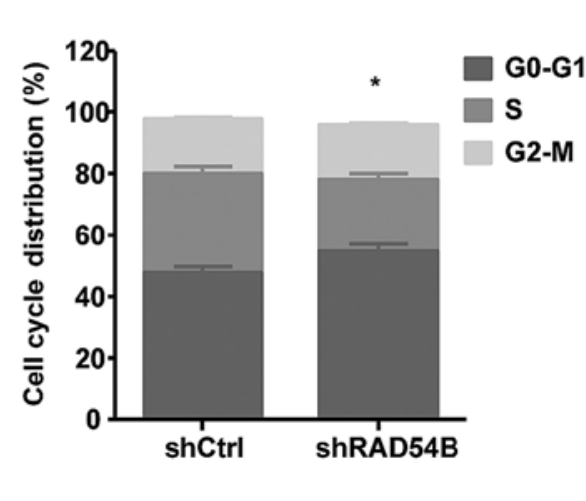

Figure 5. RAD54B inhibition induces cell cycle arrest in the G1/S phase. (A and C) BEL-7404 and SMMC-7721 cells were infected with shRAD54B and shCtrl, and the DNA content was analyzed by flow cytometry. (B and D) The percentage of cells in the G0-G1, S and G2-M phases of the cell cycle was calculated $\left(\mathrm{n}=3,{ }^{*} \mathrm{P}<0.05\right)$.

shRAD54B groups were reduced compared with the shCtrl groups from days 2 to 5 in both the BEL-7404 and SMMC7721 cell lines (Fig. 3A and B). In addition, cell counts of the shRAD54B groups were significantly lower than the shCtrl groups from days 2 to 5 (Fig. 3C and D). Similarly, the MTT assay revealed that cell growth in the shRAD54B groups was significantly suppressed compared with the shCtrl groups from days 2 to 5 (Fig. 3E and F). These results revealed that the downregulation of RAD54B inhibited cell proliferation in the BEL-7404 and SMMC-7721 cell lines.

RAD54B downregulation significantly inhibits colony formation in BEL-7404 and SMMC-7721 cells. We also analyzed the effect of shRAD54B on colony formation in the BEL-7404 and SMMC-7721 cell lines using a colony formation assay. As observed in Fig. 4A-D, there was a clear decrease in the number of colonies in the shRAD54B groups compared with the shCtrl groups. The results indicated that RAD54B downregulation significantly inhibited the colony-forming abilities of BEL-7404 and SMMC-7721 cells.

Inhibition of RAD54B induces cell cycle arrest in the G1/S phase. Flow cytometry was used to detect the cell cycle distribution and thereby determine the effects of RAD54B on liver cancer cells, including the BEL-7404 and SMMC-7721 cell lines. As shown in Fig. 5A-D, the percentage of cells in the G1 phase was significantly increased and the population of cells in the $S$ phase was significantly decreased in the shRAD54B group compared with the control group, which indicated that RAD54B downregulation induced cell cycle arrest in the G1/S phase.

RAD54B downregulation increases the apoptosis of BEL-7404 and SMMC-7721 cell lines. To further elucidate whether RAD54B affects apoptosis in hepatoma cells, we assessed the levels of apoptosis in shRAD54B and shCtrl cells. As shown in Fig. 6A-D, the number of apoptotic shRAD54B-infected cells was significantly increased compared with the shCtrl-infected cells in both BEL-7404 and SMMC-7721 cell lines. These results demonstrated that $\mathrm{RAD} 54 \mathrm{~B}$ downregulation induced apoptosis in BEL-7404 and SMMC-7721 cells.

RAD54B expression is associated with a poor prognosis in liver cancer patients. IHC staining was performed to determine the relationship between RAD54B expression and the prognosis of liver cancer patients. RAD54B protein expression was investigated in 83 samples of liver cancer tissue. The overall survival (OS) data was obtained for all 83 patients. The results revealed 
A

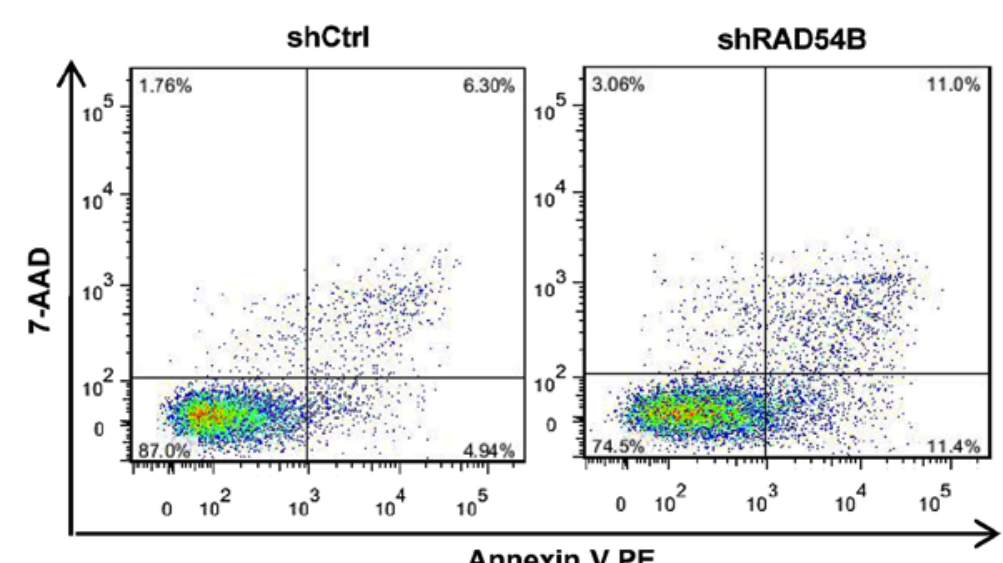

Annexin V PE

C

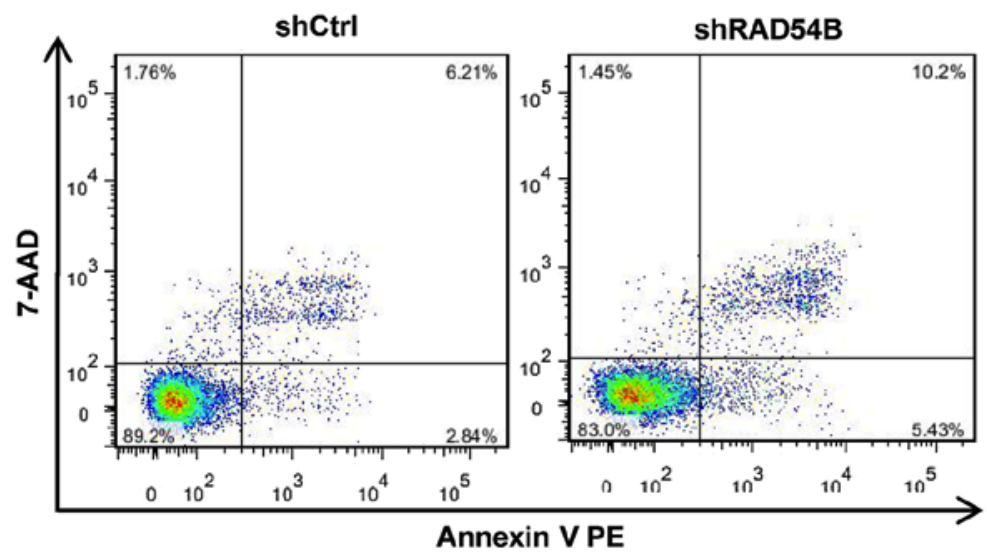

B

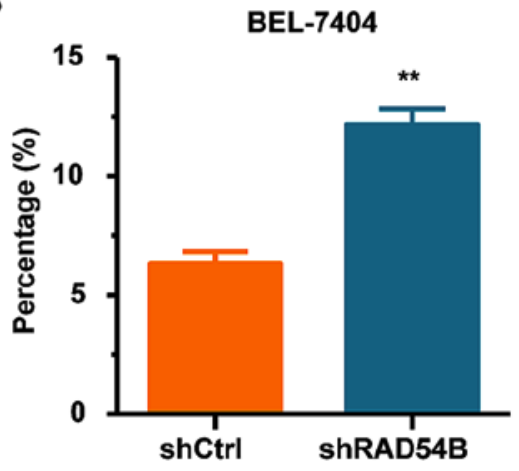

D

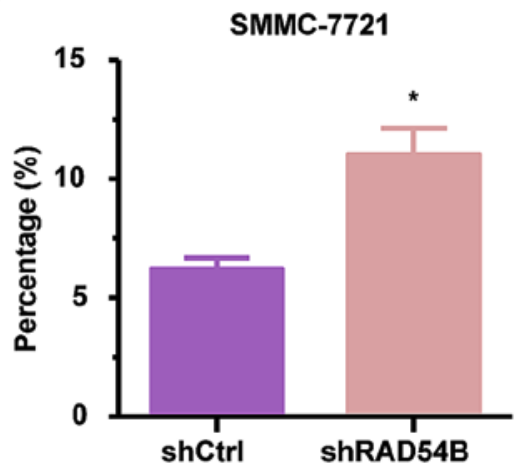

Figure 6. RAD54B downregulation induces hepatoma cell apoptosis. (A and C) The apoptosis rate of BEL-7404 and SMMC-7721 cell lines after infection with shRAD54B or shCtrl was examined by flow cytometric analysis. (B and D) The apoptotic rate of infected BEL-7404 and SMMC-7721 cells ( $\mathrm{n}=3$, "P $<0.05$, $\left.{ }^{* *} \mathrm{P}<0.01\right)$.

that $52 / 83$ samples of liver cancer were positive for nuclear staining and RAD54B protein expression, while the other 31 samples were negative (Fig. 7A). Using Kaplan-Meier survival analysis we found that the median OS time was 18.3 months for patients with positive expression, whereas the OS was 22.0 months for patients with RAD54B negative expression. Liver cancer patients with positive RAD54B expression were associated with a reduced $\mathrm{OS}(\mathrm{P}=0.02$, Fig. 7B). Collectively, these results indicated that the expression of RAD54B could be a prognostic marker for liver cancer.

\section{Discussion}

Homologous recombination (HR) is an important pathway in DSB repair; however, a number of genes and proteins associated with HR have been reported to serve a critical role in the development of cancer. Human RAD54B is an important DNA repair and recombination protein, which belongs to the DEAD-like helicase superfamily and plays an important role in the DNA damage response (DDR) by binding to doublestranded DNA and displaying ATPase activity (18-20). A number of DNA re-sequencing studies have revealed that somatic alterations in human RAD54B, including gene amplifications, mutations, homozygous deletions and single- nucleotide polymorphisms, are observed in distinct types of human cancers (21-26). The influence of RAD54B on a few types of cancer has been previously reported, however, the effect of RAD54B on hepatoma cells and its mechanism of action remain unclear. Therefore, our study focused on the role of RAD54B during the development and progression of liver cancer, and clarified that RAD54B expression was increased in liver cancer tissues, which may be associated with the poor prognosis observed in liver cancer patients. Moreover, we confirmed that RAD54B downregulation inhibited proliferation and colony formation in liver cancer cells, while inducing apoptosis. These findings indicate that RAD54B could be a potential prognostic factor for hepatoma patients.

As aforementioned, RAD54B has been previously revealed to participate in the development of some cancers, although this has not yet been demonstrated in liver cancer. Nagai et al found that RAD54B mRNA expression was elevated in the majority of colorectal cancer (CRC) tissues compared to the corresponding normal mucosa. Furthermore, CRC patients with high RAD54B expression had significantly reduced recurrence-free survival compared with patients with low RAD54B expression. Based on these results, RAD54B expression is considered to be an independent prognostic factor for distant recurrence in stage I-III 

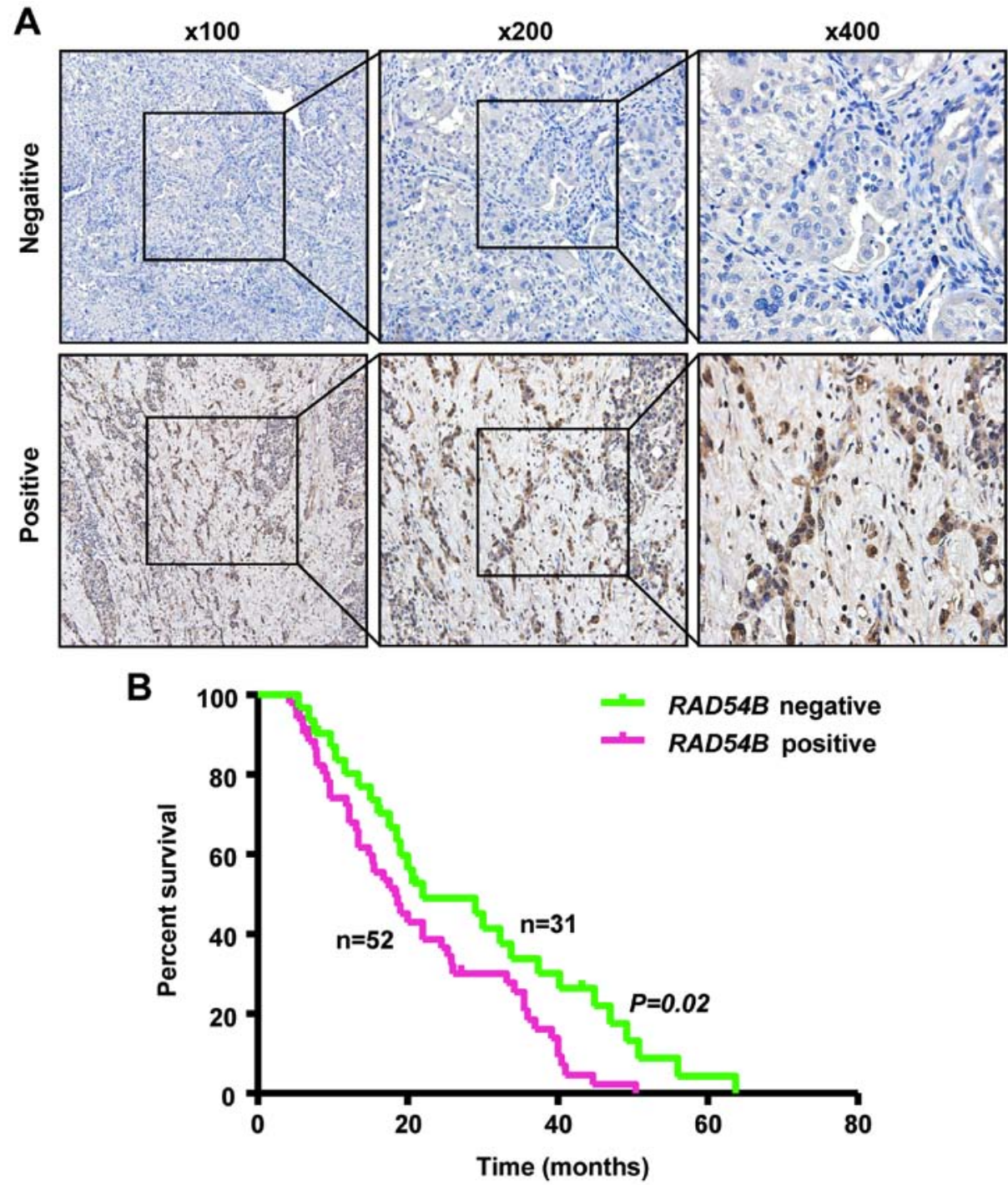

Figure 7. RAD54B expression is associated with poor prognosis in liver cancer patients. (A) IHC staining was performed to observe the expression of RAD54B in liver cancer tissues. Representative images from 52 positive expression tissues and 31 negative expression tissues are shown (magnification, $\mathrm{x} 100$, $\mathrm{x} 200$ and x400). (B) Kaplan-Meier survival analysis was used to evaluate the relationship between RAD54B expression and overall survival in liver cancer patients. The survival curve for patients with positive and negative RAD54B expression is depicted.

CRC patients (15). In addition, it has been observed that the expression of RAD54B protein was increased in patients with lung adenocarcinoma (16). Thus, we speculated that RAD54B expression could be related to the prognosis of liver cancer patients.

A number of studies have shown that other HR proteins associated with the normal repair process are closely related to the progression of liver cancer. Recently, HR proteins have been viewed as potential treatment targets for different cancers, including liver cancer. A recent study reported that gefitinib and harmine inhibited the proliferation of hepatoma cells and suppressed HR repair mediated by RAD51, which forms a protein complex with RAD54B (27). Another study performed genotype-phenotype correlation analysis of 44 human liver tissue samples, and found that RAD52 single nucleotide polymorphisms (SNPs) contributed to the susceptibility of developing hepatoma (28). Similarly, we initially found that RAD54B overexpression resulted in a poor patient prognosis in liver cancer, and that RAD54B downregulation inhibited the proliferation of hepatoma cells. However, the molecular mechanisms of this process still need to be studied further.

In conclusion, our results indicated that RAD54B inhibition suppresses proliferation and colony formation, inducing cell cycle arrest in the G1/S phase and apoptosis in liver cancer cells. Moreover, we found that RAD54B was highly expressed in hepatoma tissues, which may contribute to poor prognosis in liver cancer patients. Collectively, these results revealed that RAD54B could be a potential target for the treatment of liver cancer.

\section{Acknowledgements}

Not applicable.

\section{Funding}

The present study was supported by funding from the National Natural Sciences Fund (no. 81572315) and the Anhui Provincial Natural Science Foundation (no. 1708085QH216). 


\section{Availability of data and materials}

The datasets used during the present study are available from the corresponding author upon reasonable request.

\section{Authors' contributions}

RW and LiW participated in the design of the study, the data interpretation and manuscript drafting. YaL, YC, LeW and JL performed the experiments. QW, YG and YuL participated in the clinical sample collection. All authors read and approved the manuscript and agree to be accountable for all aspects of the research in ensuring that the accuracy or integrity of any part of the work are appropriately investigated and resolved.

\section{Ethics approval and consent to participate}

The human tissue protocol utilized in this study was approved by the Ethics Committee of Bengbu Medical College (Bengbu, China). Preoperative informed consent was obtained from each patient registered in the study, in accordance with the institutional guidelines.

\section{Patient consent for publication}

Not applicable.

\section{Competing interests}

The authors declare that they have no competing interests.

\section{References}

1. Siegel RL, Miller KD and Jemal A: Cancer Statistics, 2017. CA Cancer J Clin 67: 7-30, 2017.

2. Zuo TT, Zheng RS, Zhang SW, Zeng HM and Chen WQ: Incidence and mortality of liver cancer in China in 2011. Chin J Cancer 34: 508-513, 2015.

3. Trovato FM, Tognarelli JM, Crossey MM, Catalano D, TaylorRobinson SD and Trovato GM: Challenges of liver cancer: Future emerging tools in imaging and urinary biomarkers. World J Hepatol 7: 2664-2675, 2015.

4. O'Kane GM, Connor AA and Gallinger S: Characterization, detection, and treatment approaches for homologous recombination deficiency in cancer. Trends Mol Med 23: 1121-1137, 2017.

5. Talens F, Jalving M, Gietema JA and Van Vugt MA: Therapeutic targeting and patient selection for cancers with homologous recombination defects. Expert Opin Drug Discov 12: 565-581, 2017.

6. Kolinjivadi AM, Sannino V, de Antoni A, Técher H, Baldi G and Costanzo V: Moonlighting at replication forks - a new life for homologous recombination proteins BRCA1, BRCA2 and RAD51. FEBS Lett 591: 1083-1100, 2017.

7. Klein HL: The consequences of Rad51 overexpression for normal and tumor cells. DNA Repair (Amst) 7: 686-693, 2008.

8. Tennstedt P, Fresow R, Simon R, Marx A, Terracciano L, Petersen C, Sauter G, Dikomey E and Borgmann K: RAD51 overexpression is a negative prognostic marker for colorectal adenocarcinoma. Int J Cancer 132: 2118-2126, 2013.

9. Song H, Xia SL, Liao C, Li YL, Wang YF, Li TP and Zhao MJ: Genes encoding Pir51, Beclin 1, RbAp48 and aldolase b are up or down-regulated in human primary hepatocellular carcinoma. World J Gastroenterol 10: 509-513, 2004.

10. Obama K, Satoh S, Hamamoto R, Sakai Y, Nakamura Y and Furukawa Y: Enhanced expression of RAD51 associating protein-1 is involved in the growth of intrahepatic cholangiocarcinoma cells. Clin Cancer Res 14: 1333-1339, 2008.
11. Chandramouly G, McDevitt S, Sullivan K, Kent T, Luz A, Glickman JF, Andrake M, Skorski T and Pomerantz RT: Small-molecule disruption of RAD52 rings as a mechanism for precision medicine in BRCA-deficient cancers. Chem Biol 22: 1491-1504, 2015.

12. Huang F, Goyal N, Sullivan K, Hanamshet K, Patel M, Mazina OM, Wang CX, An WF, Spoonamore J, Metkar S, et al: Targeting BRCA1- and BRCA2-deficient cells with RAD52 small molecule inhibitors. Nucleic Acids Res 44: 4189-4199, 2016.

13. Cramer-Morales K, Nieborowska-Skorska M, Scheibner K, Padget M, Irvine DA, Sliwinski T, Haas K, Lee J, Geng H, Roy D, et al: Personalized synthetic lethality induced by targeting RAD52 in leukemias identified by gene mutation and expression profile. Blood 122: 1293-1304, 2013.

14. Wang Y, Gudikote J, Giri U, Yan J, Deng W, Ye R, Jiang W, Li N, Hobbs BP, Wang J, et al: RAD50 expression is associated with poor clinical outcomes after radiotherapy for resected non-small cell lung cancer. Clin Cancer Res 24: 341-350, 2018.

15. Nagai Y, Yamamoto $Y$, Yasuhara $T$, Hata K, Nishikawa $T$, Tanaka T, Tanaka J, Kiyomatsu T, Kawai K, Nozawa H, et al: High RAD54B expression: An independent predictor of postoperative distant recurrence in colorectal cancer patients. Oncotarget 6: 21064-21073, 2015.

16. Hwang JC, Sung WW, Tu HP, Hsieh KC, Yeh CM, Chen CJ, Tai HC, Hsu CT, Shieh GS, Chang JG, et al: The overexpression of FEN1 and RAD54B may act as independent prognostic factors of lung adenocarcinoma. PLoS One 10: e0139435, 2015.

17. Livak KJ and Schmittgen TD: Analysis of relative gene expression data using real-time quantitative PCR and the 2(-Delta Delta C(T)) Method. Methods 25: 402-408, 2001.

18. Smith CL and Peterson CL: A conserved Swi2/Snf2 ATPase motif couples ATP hydrolysis to chromatin remodeling. Mol Cell Biol 25: 5880-5892, 2005.

19. Miyagawa K, Tsuruga T, Kinomura A, Usui K, Katsura M, Tashiro S, Mishima H and Tanaka K: A role for RAD54B in homologous recombination in human cells. EMBO J 21: 175-180, 2002.

20. Sarai N, Kagawa W, Fujikawa N, Saito K, Hikiba J, Tanaka K, Miyagawa K, Kurumizaka H and Yokoyama S: Biochemical analysis of the N-terminal domain of human RAD54B. Nucleic Acids Res 36: 5441-5450, 2008.

21. Bass AJ, Thorsson V, Shmulevich I, Reynolds SM, Miller M, Bernard B, Hinoue T, Laird PW, Curtis C, Shen H, et al; Cancer Genome Atlas Research Network: Comprehensive molecular characterization of gastric adenocarcinoma. Nature 513: 202-209, 2014.

22. Ciriello G, Gatza ML, Beck AH, Wilkerson MD, Rhie SK, Pastore A, Zhang H, McLellan M, Yau C, Kandoth C, et al; TCGA Research Network: Comprehensive molecular portraits of invasive lobular breast cancer. Cell 163: 506-519, 2015.

23. Imielinski M, Berger AH, Hammerman PS, Hernandez B, Pugh TJ, Hodis E, Cho J, Suh J, Capelletti M, Sivachenko A, et al: Mapping the hallmarks of lung adenocarcinoma with massively parallel sequencing. Cell 150: 1107-1120, 2012.

24. Hodis E, Watson IR, Kryukov GV, Arold ST, Imielinski M, Theurillat JP, Nickerson E, Auclair D, Li L, Place C, et al: A landscape of driver mutations in melanoma. Cell 150: 251-263, 2012.

25. Dulak AM, Stojanov P, Peng S, Lawrence MS, Fox C, Stewart C, Bandla S, Imamura Y, Schumacher SE, Shefler E, et al: Exome and whole-genome sequencing of esophageal adenocarcinoma identifies recurrent driver events and mutational complexity. Nat Genet 45: 478-486, 2013.

26. Hiramoto T, Nakanishi T, Sumiyoshi T, Fukuda T, Matsuura S, Tauchi H, Komatsu K, Shibasaki Y, Inui H, Watatani M, et al: Mutations of a novel human RAD54 homologue, RAD54B, in primary cancer. Oncogene 18: 3422-3426, 1999.

27. Shao J, Xu Z, Peng X, Chen M, Zhu Y, Xu L, Zhu H, Yang B, Luo $\mathrm{P}$ and $\mathrm{He} \mathrm{Q}$ : Gefitinib synergizes with irinotecan to suppress hepatocellular carcinoma via antagonizing Rad51-mediated DNA-repair. PLoS One 11: e0146968, 2016.

28. Li Z, Guo Y, Zhou L, Ge Y, Wei L, Li L, Zhou C, Wei J, Yuan Q, Li J, et al: Association of a functional RAD52 genetic variant locating in a miRNA binding site with risk of HBV-related hepatocellular carcinoma. Mol Carcinog 54: 853-858, 2015.

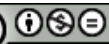

This work is licensed under a Creative Commons Attribution-NonCommercial-NoDerivatives 4.0 International (CC BY-NC-ND 4.0) License. 